\title{
Time-Resolved Imaging of Electrochemical Switching in Nanoscale Resistive Memory Elements
}

William A. Hubbard ${ }^{1}$, E. R. White ${ }^{1}$, Alexander Kerelsky ${ }^{1}$, G. Jasmin ${ }^{1}$, Jared J. Lodico ${ }^{1}$, Matthew Mecklenburg $^{2}$, and B. C. Regan ${ }^{1}$

1. Department of Physics \& Astronomy and California NanoSystems Institute, University of California, Los Angeles, CA 90095 USA

2. Center for Electron Microscopy and Microanalysis, University of Southern California, Los Angeles, CA 90089 USA

FLASH memory is reaching its scaling limit, but resistive random access memory (ReRAM) is considered a promising successor [1]. In ReRAM, metal electrodes sandwiching an insulating electrolyte form a digital memory element, where the presence or absence of a conducting path through the insulator represents one bit of information. The conducting filament is thought to form, atom-by-atom, when subject to a SET voltage applied across the electrodes, and to disintegrate when subject to a RESET voltage. We use scanning transmission electron microscopy (STEM) to image nanoscale ReRAM devices switching in situ. Operating the devices with small current limits slows the rate of filament formation and reduces confounding thermal effects, allowing us to obtain time-resolved images of filament formation and regeneration.

We fabricated $\mathrm{Pt} / \mathrm{Al}_{2} \mathrm{O}_{3} / \mathrm{Cu}$ ReRAM devices with a slant-vertical geometry. The "slant" gives good imaging access, while keeping the electrodes completely encased to ensure that conducting filaments form though the $\mathrm{Al}_{2} \mathrm{O}_{3}$ layer (as in the standard vertical geometry) and not across exposed surfaces or layer interfaces [2]. Figure 1a shows a STEM image of a pristine device, and Fig. 1b shows the same device after a 4.4 V SET bias has been applied. Applying the SET caused the current to jump to the 50 nA compliance limit as the device switched from its $\sim 10 \mathrm{G} \Omega$ OFF state to a $\sim 20 \mathrm{M} \Omega \mathrm{ON}$ state. The STEM images show that, coincident with the SET, the device developed a $5 \mathrm{~nm}$ wide $\mathrm{Cu}$ filament connecting the electrodes. After a -4V RESET, the device was cycled repeatedly between the ON and OFF states. The low current compliance regulated the rate of the switching processes so that the SET and RESET processes could be time-resolved: each was imaged over several frames. The electronic and imaging data acquired under these conditions corroborate the electrochemical model for switching [3].

Devices tested with higher current limits showed evidence of heating during SET. Figures 1c-e show STEM images of devices that were switched with SET bias voltage values comparable to those of the device in 1a-b, but with current limits ranging from $100 \mathrm{nA}$ to $700 \mathrm{nA}$ (i.e. 2 to 14 to times larger). The resulting filaments are much wider than the filaments formed during low current SETs, and the ON resistances are $<1 \mathrm{k} \Omega$. The electrodes exhibit reconfiguration and recrystallization consistent with heating to temperatures near their respective melting points. However, the programmed current limit values used are still small enough that such high temperatures are unexpected. RESETs following such large-current SETs could require more than $1 \mathrm{~mA}$, which often resulted in device failure (Fig. 1f).

Using the larger compliance currents decreases the ON-state resistance $R_{\mathrm{ON}}$, which allows transient currents larger than the compliance current limit to flow. These currents are sourced by stray capacitance $C$ in the circuit, and can Joule-heat the device by hundreds of kelvins. A simplified circuit model of the ReRAM biasing setup is shown in Fig. 2. The time constant for the system is $\tau \sim C$ RoN, 
and the maximum current $I \simeq \mathrm{Q} / \tau \simeq C V / \tau \simeq V / R$ ON. Therefore, the maximum power through a device during an $\mathrm{ON}$ switch is $I V=V^{2} / R_{\mathrm{ON}}$. For a device with a $R_{\mathrm{ON}}=1 \mathrm{k} \Omega$, the $\gtrsim 1 \mathrm{~mW}$ SET heats the device above a thermal threshold. In comparison a $R_{\mathrm{ON}}=10 \mathrm{M} \Omega$ device switches with $100 \mathrm{nW}$ and remains near room temperature. Small compliance currents thus enable time-resolved imaging of ReRAM cycling in situ.

\section{References:}

[1] I. Valov et al, Nanotechnology 22 (2011), p. 289502

[2] W. Hubbard et al, Microscopy and Microanalysis 20 (2014), p. 1550-1551

[3] S. Menzel et al, Physical Chemistry Chemical Physics 20 (2013), p. 6945

[4] This work has been supported by FAME, one of six centers of STARnet, a Semiconductor Research Corporation program sponsored by MARCO and DARPA, and by National Science Foundation award DMR-1206849. The authors acknowledge the use of instruments at the Electron Imaging Center for NanoMachines supported by NIH 1S10RR23057 and the CNSI at UCLA.
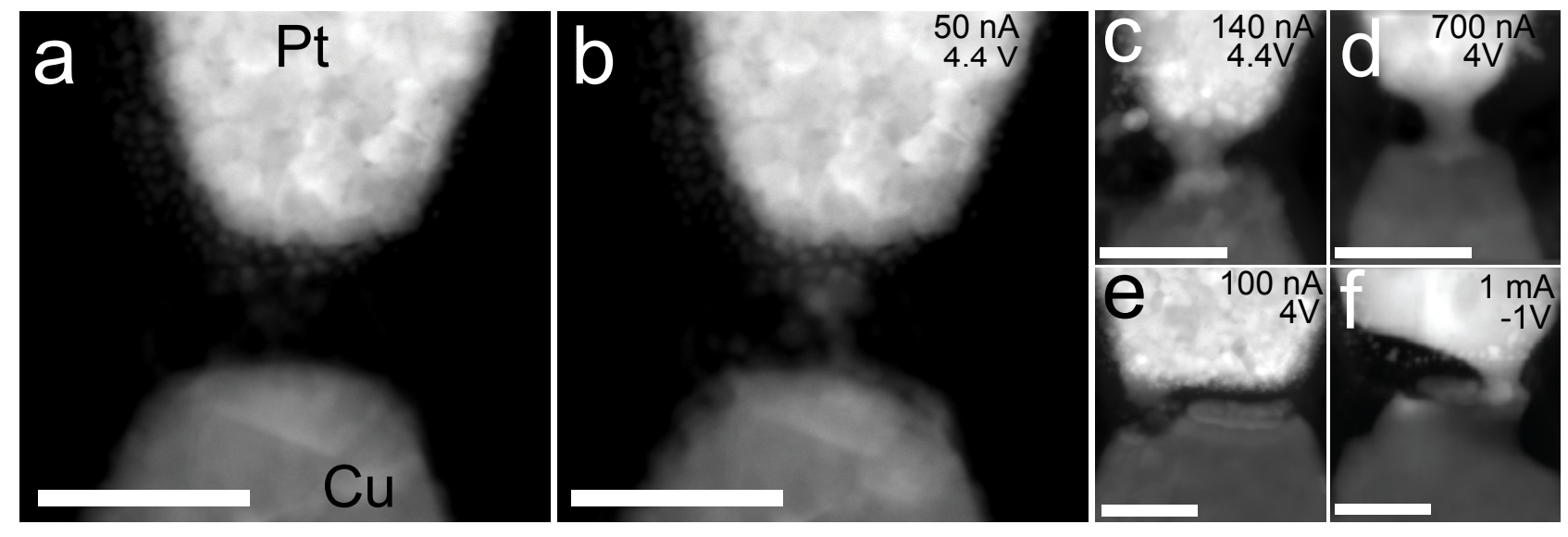

Figure 1. STEM images of a slant-vertical ReRAM device before (a) and after (b) its first SET (voltage and current limit shown). Similar devices (c-e) that were switched with larger current limits produced burlier connections with poorer cycling endurance. For instance, f shows the e device after a RESET attempt failed to change the device state to OFF. The scale bar in all images is $50 \mathrm{~nm}$.

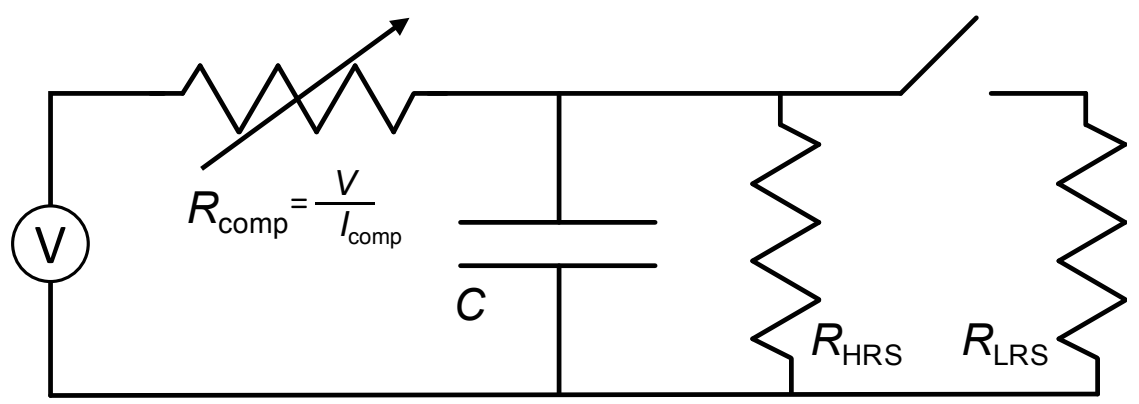

Figure 2. Circuit model of ReRAM device biasing. The resistance $R_{\text {comp }}$ is determined by the programmed current compliance $I_{\text {comp }}$. The ON state device resistance $R_{\mathrm{ON}}$, a function of $I_{\text {comp }}$, decreases rapidly once $I_{\text {comp }}$ exceeds a thermal threshold value that in our devices is $\leqslant 100 \mathrm{nA}$. 